\title{
Tetracycline and toxicity induced
}

\begin{abstract}
Tetracyclines have been used extensively as broad-spectrum antibiotics. They are effective against a wide variety of human diseases and animal caused by different microbial pathogens. The mode of Tetracycline action is reversibly inhibiting bacterial protein synthesis by binding with the prokaryotic $30 \mathrm{~S}$ ribosomal subunit that prevents the association of aminoacyl-tRNA with the ribosomal target site and subsequently inhibits protein synthesis. Toxicity of tetracycline antibiotics was proven, by many studies, in which induction of fatty liver, acute pancreatitis, severe hepato-nephrotoxicity, contribute to the development and severity of microvesicular steatosis, increase in the triglycerides, AST, ALT, bilirubin, urea, creatinine and gamma-globulin levels. Histological analysis of liver samples of tetracycline-treated rats revealed high vacuolation of the cytoplasm of hepatic cells, sinusoidal dilation, hepatocellular necrosis and disappearance of the cell membrane in some hepatocytes. Tetracycline can also cause chromosomal abnormalities as well as interfere with male fertility.
\end{abstract}

Keywords: tetracycline antibiotics, antibiotic toxicity
Volume 10 Issue 4 - 2019

\section{Omhashem EA Abdel-Gelil, Samira R Mansour \\ Department of Zoology, Suez Canal University, Egypt}

Correspondence: Samira R Mansour, Professor of Microbiology, Botany Department, Faculty of Science, Suez Canal University, Ismailia, Egypt, Tel +201003686076, Fax +20 643230416 ,

Emailsamirarmansour@hotmail.com, Samira_lbrahim@ science_suezcanal.edu.eg

Received: May 28, 2019 | Published: July 08, 2019

\section{Introduction}

Antibiotic simply is a type of medication used to treat bacterial infections. It is a substance produced by one microorganism that selectively inhibits the growth of pathogenic microbes. It is considered as secondary metabolites that enhanced the microbial defense against biotic and abiotic stresses. Treatment of bacterial infection using antibiotics is either by inhibiting the bacterial growth (Bacteriostatic) or destroys the bacterial cell (Bactericidal). Although antibiotics are useful in a wide variety of infections, it is important to realize that antibiotics only treat bacterial infections rather than viral and fungal infections. There are two types of antibiotics, Broad Spectrum Antibiotics that affect several types of bacterial infections and they are normally used where the specific type of the microorganism is unknown. Meanwhile, Narrow spectrum antibiotics, are those used to inhibit or kill particular organisms. Tetracycline and its semi synthetic derivatives are among the broad spectrum antibiotics.

\section{Classification of antibiotics}

There are different classes of antibiotics depending on the bases of their chemical structures and the action mechanism.

\section{Depending on their chemical structures}

Antibiotics, in use up till now, are diverse fundamental groups in which addition or removing of chemical groups from the core structure produces numerous active members of the mother group. Based on the classification, done by Gordon ${ }^{1}$ antibiotics can be classified into Beta-lactam antibiotics, Macrolides, Lincosamides, Aminoglycosides, Tetracyclines, Polypeptides, Sulfonamides, and Fluoroquinolones. Therefore, Tetracyclines are considered among the main groups of antibiotics which can natural or semisynthetic produced. Chlortetracycline and Oxytetracycline, first members of Tetracycline group that discovered in the late 1940s, were products of actinobacteria of genus Streptomyces aureofaciens and S. rimosus, respectively. However, the most recently discovered Tetracyclines are the semisynthetic group referred to as glycylcyclines.

\section{Depending on their mechanisms}

There are three different mechanisms on which antibiotics work on the bacterial cell structures. The antibiotics can inhibit cell wall synthesis, inhibit protein synthesis involved in the biosynthesis of cytoplasmic membrane/or disruption of bacterial membrane structure (polymyxins), interference with nucleic acid synthesis (quinolones), and in sequence, inhibit certain metabolic pathways (Trimethoprim, Primsulfa, and Methoxazole).

Antibiotics that involved in interference with cell wall synthesis ( $\beta$-lactam antibiotics) are considered as Bactericidal antibiotics. They kill bacteria by inhibiting cell wall synthesis; inhibit the cell wall enzyme MurA (Phosphonic acids) and another group that can inhibit the transglycosylation and transpeptidation reactions that are required for completion of the peptidoglycan chain (Glycopeptides). Meanwhile, antibiotics that are targeting the cytoplasmic membrane, the second structure lying behind the cell wall of the bacterial cell, are targeting phospholipids of the bacterial cell membrane or altering their properties. Meanwhile, DNA targeting antibiotics, known as Bacteriostatic antibiotics, are inhibiting DNA synthesis, RNA synthesis or/and protein synthesis. ${ }^{2,3}$

The world consumption of antibiotics has risen drastically in the last decade, especially those that are considered to be safe and have many favorable properties. Tetracyclines (TCs) are among these antibiotics and they are the third most consumed antibiotic, after penicillin and quinolones (Borghi and $\mathrm{Palma}^{4}$ ). TCs are a family of Bacteriostatic drugs that inhibit or delay bacterial growth and replication. The mode of action, of these antibiotics, is to inhibit protein synthesis by preventing the attachment of aminoacyl-tRNA to the ribosomal acceptor (A) site. ${ }^{5}$ Therefore; their main role is inhibiting the growth of Gram-positive and Gram-negative bacteria by binding to their ribosomes and stopping protein synthesis and in sequence the whole activity of the bacterial cell.

TCs drugs have gained wide acceptance in the third world countries because of its safety, easy accessibility, availability and cost-effectiveness as well as their multiuse. They can be used as anti- 
apoptotic and anti-inflammatory where several studies demonstrate such effects in the context of neuroprotection. ${ }^{6,7}$ In addition, a subclinical dose of tetracycline is likely to be used to protect the heart from ischemic injury and be of therapeutic value in suppressing the onset of infarction caused by myocardial ischaemia, ${ }^{8}$ wound healing actions and antiaging since they can inhibit enzyme systems involved in connective tissue and collagen breakdown. ${ }^{9}$ Although they are generally regarded as relatively nontoxic drugs they produce a fairly large number of side effects. Some of them are positive, and others are life-threatening under certain circumstances.

\section{Toxicity and side effects of tetracycline antibiotic}

The most commonly reported side effect of oral tetracycline is gastrointestinal distress, such as nausea and esophagitis. ${ }^{10}$ The second most common side effect of tetracyclines is skin reaction (rash, pruritus, and photosensitivity). Tetracyclines can also lead to permanent discoloration of teeth in children whose teeth are developing, as the drug incorporates into tissues that are calcifying at the time of their administration leading to yellow or gray to brown discoloration of both the primary and permanent dentitions. ${ }^{11}$

Toxicity of TCs has proven by long use of this antibiotic or as high dose responses in which kidney and liver are seriously affected. ${ }^{12-14}$ In their studies, they concluded that the renal side effects of tetracycline showed an increase in Fanconi's syndrome and blood urine nitrogen (BUN). In patients with pre-existing renal impairment, tetracycline may cause azotemia, hyperphosphatemia, and acidosis. In meantime, many other studies revealed that TCs induced a fatty liver in rats by inhibiting mitochondrial $\beta$-oxidation and microsomal triglyceride transfer protein which in sequence inhabit the hepatic secretion of triglyceride. ${ }^{15,16}$ In addition TCs side effects, they may lead to an increase in the triglycerides, LDL-cholesterol, AST, ALT, bilirubin, urea, creatinine and gamma-globulin levels. ${ }^{17}$ Tetracycline may also cause acute pancreatitis which may result in increased serum amylase activity. ${ }^{18}$ Antioxidant enzymes, superoxide dismutase, glutathione peroxidase, glutathione reductase, and catalase, showed also a small but significant decrease in the pancreas of the TCs-treated rats..$^{18,19}$

TCs may also affect the mortality rate and consider as a source of severe hepato-nephrotoxicity. These were demonstrated by biochemical analysis of serum including transferases, alkaline phosphatase, total protein and glucose. ${ }^{20}$ In parallel, TCs inhibit the mitochondrial oxidation of fatty acids in mice and in man. ${ }^{17,21}$ This effect may contribute to the development and severity of microvascular steatosis observed after high doses of this antibiotic in man. Tetracycline derivatives could induce folate-deficient megaloblastic anemia when long term treatment applied. Meanwhile, treatment with Oxytetracycline has been incriminated as a cause of thrombocytopenic purpura (Kounis, 1975). In parallel, renal dysfunction may occur due to a high dose or long treatment period. ${ }^{17,22}$

Histological analysis of liver samples of tetracycline-treated rats revealed high vacuolation of the cytoplasm of hepatic cells, sinusoidal dilation, hepatocellular necrosis and disappearance of the cell membrane in some hepatocytes. TCs are also affect the reproductive organ in human been The long-term effect of these antibiotics on male reproductive tract development led to long-lasting effects on reproductive organs and spermatozoa which in sequence exhibited significant reduction of sperm viability ${ }^{23,24}$ and reduction in testosterone level. ${ }^{25} \mathrm{TCs}$ also caused significant decrease in the relative weights of the testis, epididymis, seminal vesicles, and reduction in the epididymal sperm motility, percentage of live spermatozoa, sperm count, and an increase in abnormal sperm morphology, as well as induction of adverse histopathologic changes in the testes. ${ }^{26}$

\section{Conclusion}

Tetracyclines, such as tetracycline, chlortetracycline, doxycycline, or minocycline, are oral wide spectra bacteriostatic antibiotics. They act upon the conserved sequences of the 16S rRNA of the 30S ribosomal subunit to prevent binding of t-RNA to the A site. They can be used to cure different infections. Although they are generally regarded as relatively nontoxic drugs they produce a fairly large number of side effects. Some of them are positive, and others are lifethreatening under certain circumstances. Studies on TCs proved to be toxic to the human when they used for a long period. A high dose of TCs orally administrated also showed toxic effects which affect many human body organs including liver and kidney. Administration of tetracycline also showed significant reduced sperm count, sperm motility, percent normal morphology, and percent live sperm. In general, we can say that the continuous use of TC in a random way and in high dose is not safe and more attention should be paid to give awareness for the hazard and negative effect of blind uses of TCs on the human and animal health level.

\section{Acknowledgments}

Authors express their deep thanks to all colleagues who encourage and help to finish up this mini review.

\section{Conflicts of interest}

Author declares there are no conflicts of interest towards this article.

\section{References}

1. Gordon. Introduction to antimicrobial drugs. Purdue Research Foundation. 1996.

2. Opperman TJ, Kwasny SM, Li JB, et al. DNA targeting as a likely mechanism underlying the antibacterial activity of synthetic bis-indole antibiotics. Antimicrob Agents Chemother. 2016;60(12):7067-7076.

3. Kapoor G, Saigal S, Elongavan A. Action and resistance mechanisms of antibiotics: A guide for clinicians. J Anaesthesiol Clin Pharmacol. 2017;33(3):300-305.

4. Borghi AA, Palma MSA. Tetracycline: production, waste treatment, and environmental impact assessment. Braz J Pharm Sci. 2014;50(1):25-40.

5. Chopra I, Roberts M. Tetracycline antibiotics: Mode of action, applications, molecular biology, and epidemiology of bacterial resistance. Microbiol Mol Biol Rev. 2001;65(2):232-260.

6. Kraus RL, Pasieczny R, Lariosa-Willingham K, et al. Antioxidant properties of minocycline neuroprotection in an oxidative stress assay and direct radical-scavenging activity. J Neurochem. 2005;94(3):819-827.

7. Ryu JK, Choi HB, McLarnon JG. Combined minocycline plus pyruvate treatment enhances the effects of each agent to inhibit inflammation, oxidative damage and neuronal loss in an excitotoxic animal model of Huntington's disease. Neuroscience. 2006;141(4):1835-1848.

8. Kagawa N, Senbonmatsu TA, Satoh K, et al. Tetracycline protects the myocardium against ischemic injury. Front Biosci. 2005;10:608-619.

9. Golub LM, Lee HM, Ryan ME, et al. Tetracyclines inhibit connective tissue breakdown by multiple non-antimicrobial mechanisms. Adv Dent Res. 1998;12(2):12-26. 
10. Smith K, Leyden JJ. Safety of doxycycline and minocycline: a systematic review. Clin Ther. 2005;27(9):1329-1342.

11. Antonini LG, Luder HU. Discoloration of teeth from tetracyclines - even today? Schweiz Monatsschr Zahnmed. 2011;121(5):414-431.

12. Silva Machado AL, Haberbeck Brandão AA, de Oliveira Monteiro CM, et al. Influence of tetracycline in the hepatic and renal development of rat's offspring. Braz Arch Biol Technol. 2003;46(1):47-51.

13. Drug.com. Doxycycline Side Effects. 2019.

14. Szalowska E, van der Burg B, Man HY, et al. Model Steatogenic Compounds (Amiodarone, Valproic Acid, and Tetracycline) Alter Lipid Metabolism by Different Mechanisms in Mouse Liver Slices. PLoS One. 2014;9(1):e86795.

15. Fréneaux E, Labbe $G$, Letteron $P$, et al. Inhibition of the Mitochondrial Oxidation of Fatty Acids by Tetracycline in mice and in man: Possible role in microvesicular steatosis induced by this antibiotic. Hepatology. 1988;8(5):1056-1062.

16. Letteron P, Angela Sutton, Abdellah Mansouri, et al. Inhibition of microsomal triglyceride transfer protein: another mechanism for druginduced steatosis in mice. Hepatology. 2003;38(1):133-140.

17. Shabana MB, Hania M Ibrahim, Soheir EM, et al. Influence of rifampicin and tetracycline administration on some biochemical and histological parameters in albino rats. The Journal of Basic \& Applied Zoology. 2012;65(5):299-308.

18. Zendehbad B, Alipour A, Zendehbad H, et al. Effect of tetracycline administration on serum amylase activity in calves. SpringerPlus. $2013 ; 2: 330$.
19. Elmore MF, Rogge JD. Tetracycline-induced pancreatitis. Am J Gastroenterol. 1991;86(11):1669-1671.

20. Nada SA, Mahmoud S Arbid. Protective Effect of Panax ginseng Against Tetracycline Toxicity in Rats. J of ginseng research. 2004;24(2):93-98.

21. Chatzispyrou IA, Held NM, Mouchiroud L, et al. Tetracycline antibiotics impair mitochondrial function and its experimental use confounds research. Cancer Res. 2015;75(21):4446-4449.

22. Rosenberg PH, Wahlström T. Renal and hepatic toxicity of methoxyflurane in combination with tetracycline or oxytetracycline treatment in rats. Acta Pharmacol Toxicol (Copenh). 1974;34(1):46-57.

23. Zeh JA, Bonilla MM, Adrian AJ, et al. From father to son: transgenerational effect of tetracycline on sperm viability. Sci Rep. 2012;2:375.

24. Elzeinova F, LukasDed P, Kubatova A, et al. Adverse effect of tetracycline and doxycycline on testicular tissue and sperm parameters in CD1 outbred mice. Exp Toxicol Pathol. 2013;65(6):911-917.

25. Popoola OB, Adefule AK, Ajayi RT, et al. Effects of tetracycline on testis and testosterone level in adult male Wistar rats. Scholarly J Med. 2014;4(1):4-10.

26. Oyedeji KO, Bolarinwa AF, Dare A. Effect of Tetracycline on Reproductive Functions in Male Albino Rats. IOSR Journal of Dental and Medical Sciences. 2013;3(5):55-60. 